\title{
Efficient Mechanism for Aggregate Demand Prediction in the Smart Grid
}

\author{
Péter Egri ${ }^{1}$ and József Váncza ${ }^{1,2}$ \\ 1 Fraunhofer Project Center for Production Management and Informatics \\ Institute for Computer Science and Control \\ Hungarian Academy of Sciences \\ Kende u. 13-17, 1111 Budapest, Hungary \\ 2 Department of Manufacturing Science and Technology \\ Budapest University of Technology and Economics \\ H-1111 Budapest, Egry József u. 1., Hungary \\ \{egri, vancza\}@sztaki.mta.hu
}

\begin{abstract}
This paper studies an aggregate demand prediction problem relevant in smart grids. In our model, an aggregator agent is responsible for eliciting the demand forecasts of a number of self-interested home agents and purchasing electricity for them. Forecasts are given in form of probability distributions, and generating them incurs costs proportional to their precision. The paper presents a novel scoring rule based mechanism which not only makes the agents interested in reporting truthfully, but also inspires them to achieve the socially optimal forecast precision. Hence, the aggregator agent is then able to optimise the total expected cost of electricity supply. Therefore the mechanism becomes efficient, contrarily to prior works in this field. Empirical studies show that it is beneficial to join to the mechanism compared to purchasing electricity directly from the market, even if the mechanism consists only of a few agents.
\end{abstract}

Keywords: Smart grid, distributed optimisation, information aggregation, mechanism design, scoring rules

\section{Introduction}

In the energy sector, supplies are meticulously provided so as to meet projected loads. On the one hand, when actual demand exceeds supply, the response is to use increasingly expensive energy generation sources, and, in the last resort, to decrease load (e.g., through brownouts and blackouts). On the other hand, overproduction of energy incurs severe extra costs, too. The resolution relies heavily on multiplexing a set of loads in order to smooth the aggregate demand which is formed from information provided by local individual users of energy [1]. This coupling of energy and information flows is the basis of smart grids.

The paper discusses an aggregate demand prediction model where self-interested home agents can produce electricity demand forecasts at a certain cost. 
The independent agents report the forecasts to an aggregator agent that is responsible for meeting the actual total demand. While the system as a whole could operate most efficiently if the home agents would invest into making precise enough forecasts and share this information truthfully with the aggregator, in a system of self-interested agents there is no warranty for such a behaviour. Why would not take any home agent a free rider's stance and neglect forecasting? And why would it report its unbiased private forecast when it wants to make sure its demand will be met by the electricity supply? How could agents be made interested in putting effort into generating their own, sufficiently precise demand forecasts? What an incentive system could warrant truthful communication of these forecast information? In general, can any coordination mechanism facilitate the better (i.e., cheaper, more profitable, more stable) behaviour of the system as a whole than the simple aggregation of the individual operations? Why would it pay off for any agent to participate in such a system instead of going for the satisfaction of its own demand directly?

This problem setting is relevant in any domain where demand and supply has to be matched by autonomous partners who have asymmetric information and responsibilities, and where the satisfaction of aggregate, uncertain demand may incur lower costs (or higher profits) than meeting individual demands apiece. For instance, it may mitigate risks implied by market volatility as well as decrease total production and logistics costs in supply networks where a supplier is serving different retailers of the same product [2].

We investigate the above issues by means of the apparatus of mechanism design. Mechanism design, also considered inverse game theory, has a specific engineering perspective: it applies the model of non-cooperative games with agents having incomplete information, and investigates how the private information influencing the other agents' utilities can be elicited [3]. Thanks to this generic approach, beyond elaborating a complex - communication, decision and financial transaction - mechanism we can also prove some key properties of this inherently distributed system that are prerequisites of any real application.

The remainder of the paper is organised as follows. In the next section we review related works, then in Section 3 present the demand prediction and decision making model, and derive its optimal solution in a cooperative, social welfare maximising setting. In Section 4 the proposed mechanism is presented together with the proofs of its main properties in the non-cooperative case. Numerical illustrations of the system behaviour is shown in Section 5. Finally, the paper concludes in Section 6.

\section{Literature Review}

The information elicitation or prediction mechanism design problem, which has recently come into the focus also of the multiagent research, consists of several agents with some private information about the probability of some stochastic future event, and a centre whose goal is to obtain and aggregate the dispersed information $[4,5]$. There are two different kinds of such models: the peer predic- 
tion systems and the prediction markets. In the former one, the outcome (event) is subjective or unmeasurable, therefore it cannot be used as a basis for evaluating the forecasts posteriorly. In such cases, the predictions of the agents can be compared to other agents' forecasts only.

In prediction markets however, which are in our main focus from now on, there is always a clear, objective outcome. Such problems can be handled by applying the so-called strictly proper scoring rules that we briefly define here. Let us assume a set $D$ of possible events, and $\mathcal{P}$, a class of probability measures over them. A scoring rule $S: \mathcal{P} \times D \rightarrow \mathbb{R}$ is called strictly proper, if whenever an event $\xi \in D$ is drawn from the distribution $\theta \in \mathcal{P}$, then for any other $\hat{\theta} \neq$ $\theta: \mathbb{E}_{\theta}[S(\theta, \xi)]<\mathbb{E}_{\theta}[S(\hat{\theta}, \xi)]$. With other words, the score can be minimised (in expectation), if it is parametrised with the real distribution of the stochastic event. ${ }^{3}$ Well-studied examples for strictly proper scoring rules are the quadratic, the spherical and the logarithmic rules, see e.g., [4]. Applying such rules to the information elicitation problem is straightforward: if the agent with the private information is penalized proportionally to a proper score, it becomes interested in creating and providing as good a forecast as possible.

Note that it is implicitly assumed in these models that the forecast can be generated free of charge; when generating or improving the forecast involves some cost, truthful mechanisms may not exist in general [4]. However, there exist some cases when costly forecasts can be successfully included into the model. For example, in [6] several agents can provide forecasts for the same stochastic variable at different costs, and the authors present a two-stage mechanism including a reverse second-price auction for solving the information elicitation problem in this case.

Apart from the costly forecast generation, there are several other extensions of the information elicitation problem. In some models, the agents have interests in the decision of the centre, therefore they might disclose false information in order to manipulate the decision maker. Such situation is considered e.g., in [2], where the logistic decisions of a supplier can cause shortages at the retailers, which affects their profits. Further difficulties occur, when the objective function of the agents are not known exactly, or when the agents can manipulate the outcome and therefore influence the evaluation of their reported forecasts.

Information elicitation problems appear in smart grids, which intend to combine modern communication technology with the electricity network. In order to level fluctuations and optimise the generation, distribution and utilisation of electricity, the recent advances in hardware technology should be supplemented with novel software support of automation and control, as well as business models [7]. The so-called "smart meters" for example, that measure not only the electricity usage, but also record the time of the consumption, enable the introduction of time-differentiated pricing. Exploiting the price-responsive demand, this instrument can be applied as a tool for aligning the demand with the supply of fluctuating renewable energy sources (e.g., wind, solar) in the smart grids. The bidirectional communication also enables that utility companies can collect

\footnotetext{
${ }^{3}$ Note that in contrast to the usual notation, for convenience, we minimize the score.
} 
and analyse more detailed and precise information generated by agent-based forecasting at household level [8].

In [9] an aggregate demand prediction model is presented for the smart grid, where an aggregator agent elicits consumption forecasts from the home agents and purchases electricity for them. The authors present a scoring rule based mechanism that fairly distributes the savings among the agents, and prove its individual rationality, incentive compatibility and ex ante weak budget balance properties (for formal definitions, see later). However, incentive compatibility in that paper relates only to the truthful reporting of forecasts, their precision is usually not globally optimal, therefore the mechanism is not efficient. In addition, the mechanism has to artificially limit the accepted precision from the agents, thus even the optimal solution could be excluded.

A structurally similar model for supply networks is presented in [10]. In that paper a supplier collects demand forecasts from retailers, and provides a Vendor Managed Inventory (VMI) service for them. The model completely disregards the costs of forecasting, and presents a mechanism that is incentive compatible and efficient, but individual rationality is not discussed.

In general, the operations research literature provides several analytical models for optimisation of decision making in the above setting. The model in this paper applies a specialised version of the single-period stochastic lot-sizing problem, the so-called newsvendor model [11]. Since this assumes already given forecasts, the costly forecasting should be included into the model by comparing the cost and the benefit of generating an estimate with a given precision. For this problem, the value of information (VOI) approach can be applied [12].

It is not unprecedented in the literature to apply coordination contracts in the electricity industry originally developed for supply chain inventory management. In [13] different contractual forms and market structures are investigated for dampening the double marginalisation effect, i.e., when the rational decisions of self-interested decision makers lead to a non-Pareto optimal equilibrium. The problem is illustrated through a case study of the Spanish electricity market, where the demand can be fulfilled either from the futures (forward) market in advance, or promptly from the spot (balancing) market. In that paper, the demand is not stochastic but price-dependent, therefore the price can be used to balance demand with supply.

A related study can be found in [14], which focuses on the energy resources instead of consumption. The authors present a model and incentive mechanism for aggregating electricity production from distributed energy resources. In their model the estimation is characterised by the expected value only, which is later compared to the realised production, therefore no scoring rules are required.

In this paper we consider a similar model as in [9], but contrarily, our mechanism is efficient, does not bind the accepted precision, and inspires the home agents to generate the optimally precise forecasts. The presented mechanism is based on the scoring rule introduced in [10], but it is extended to consider the costly forecast generation. Furthermore, we prove additional properties of the mechanism in the discussed model like individual rationality and ex ante strong 
budget balance. Since the proposed transfer function of agent $i$ does not depend on other agents' forecasts or demands, it evaluates only the correctness of agent $i$ 's estimation, in this sense it can be considered fair, and provides the privacy of the consumption data.

\section{Model}

We assume $n$ home agents and an aggregator agent who collects forecasts about the future consumption of the homes, and purchases electricity for them (see Fig. 1). Each day is divided into a number of periods, and for each period a home agent can generate demand forecast at a cost proportional to the forecast precision. From now on, we consider only one period, since the problems for different periods are independent from each other.

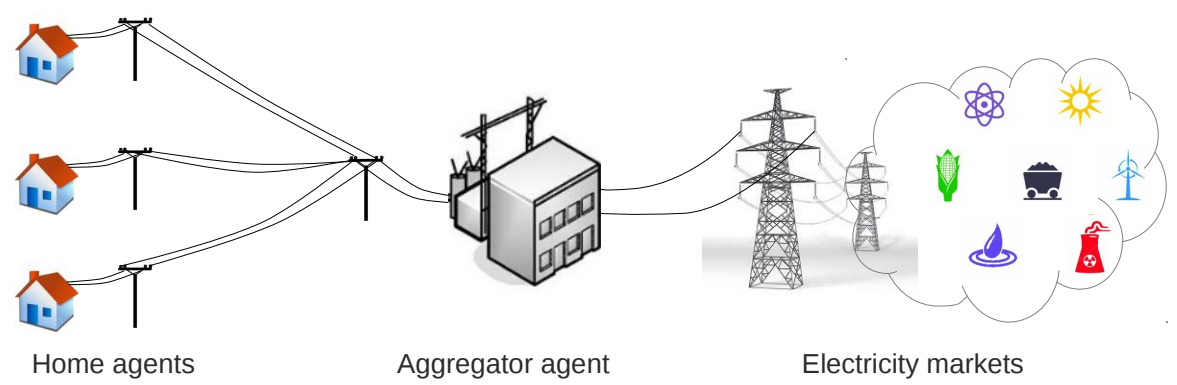

Fig. 1. Electricity network.

We assume that the forecast of agent $i \in[1, n]$ is represented by a normal distribution with mean $m_{i}$ and standard deviation $\sigma_{i}$. This forecast can be generated at price $p\left(\sigma_{i}\right)=\alpha / \sigma_{i}^{2}$, where $1 / \sigma_{i}^{2}$ is the so-called precision of the forecast, and $\alpha>0$ is a constant. Note that we assume that the price function is the same for all agents.

It is also assumed that the consumptions at the homes are independent from each other, therefore the aggregate forecast of the total consumption will also be normally distributed with mean $m=\sum_{i=1}^{n} m_{i}$ and $\sigma=\sqrt{\sum_{i=1}^{n} \sigma_{i}^{2}}$.

The aggregator agent decides about the purchase quantity $q$ that is bought on the forward market at price $c$. During the period, agent $i$ consumes $\xi_{i}$ electricity, and if the total realized consumption $\xi=\sum_{i=1}^{n} \xi_{i}$ is less or equal than the quantity bought on the forward market, the aggregator can provide the necessary electricity. However, if $\xi>q$, the aggregator has to buy additional electricity on the balancing market at buy price b. On the other hand, if $\xi<q$, the surplus can be sold there at sell price $s$. It is natural to assume the following relation between the different prices: $b>c>s$.

In the following two subsections we study the social welfare maximising solution in the centralised model considering cooperative agents. The resulted de- 
cision problem has two stages: in the first stage the optimal forecast precisions, while in the second stage the optimal purchase quantity is computed. We solve the problem in a backward induction manner: firstly, we determine the optimal $q$ assuming a given forecast with a special form of the newsvendor model, then we derive the optimal forecast precision.

\subsection{Optimal Purchase Quantity in the Cooperative Case}

In this subsection we consider the normally distributed total consumption $\xi \sim$ $\mathcal{N}(m, \sigma)$, and denote its probability and cumulative distribution functions with $\phi$ and $\Phi$, respectively. If the aggregator purchases quantity $q$, its resulted revenue will be $-c q-b \max (\xi-q, 0)+s \max (q-\xi, 0)$, i.e., the payment for the electricity on the forward market and the (negative or positive) payment of matching supply and demand on the balancing market. The expected value of this, which we call valuation, can be expressed in the following form:

$$
\begin{aligned}
v(q)= & -c q-b \mathbb{E}[\max (\xi-q, 0)]+s \mathbb{E}[\max (q-\xi, 0)] \\
= & -c q-b \int_{q}^{\infty}(x-q) \phi(x) d x+s \int_{-\infty}^{q}(q-x) \phi(x) d x \\
= & -c q-b\left(\int_{q}^{\infty} x \phi(x) d x-q \int_{q}^{\infty} \phi(x) d x\right) \\
& +s\left(q \int_{-\infty}^{q} \phi(x) d x-\int_{-\infty}^{q} x \phi(x) d x\right) \\
= & -c q-b\left(m-\int_{-\infty}^{q} x \phi(x) d x-q(1-\Phi(x))\right) \\
& +s\left(q \Phi(x)-\int_{-\infty}^{q} x \phi(x) d x\right) \\
= & -c q+b(q-m)+(b-s)\left(\int_{-\infty}^{q} x \phi(x) d x-q \Phi(q)\right) .
\end{aligned}
$$

Since the valuation function is concave $\left(v^{\prime \prime}(q)=-(b-s) \phi(q) \leq 0\right)$, the optimal $q^{*}$ can be determined by the first derivative test

$$
v^{\prime}\left(q^{*}\right)=b-c-(b-s) \Phi\left(q^{*}\right)=0,
$$

which results in

$$
q^{*}=\Phi^{-1}\left(\frac{b-c}{b-s}\right)=m+\sigma \sqrt{2} \operatorname{erf}^{-1}\left(\frac{b-2 c+s}{b-s}\right),
$$

where

$$
\operatorname{erf}(x)=\frac{2}{\sqrt{\pi}} \int_{0}^{x} e^{-t^{2}} d t
$$


is the Gauss error function and $\operatorname{erf}^{-1}(x)$ denotes its inverse. The valuation using the optimal purchase quantity can then be expressed by substituting (3) into (1) as

$$
v^{*}(\sigma):=v\left(q^{*}\right)=-c m-\sigma K_{c b s},
$$

where

$$
K_{c b s}=\frac{(b-s) e^{-\left(\operatorname{erf}^{-1}\left(\frac{b-2 c+s}{b-s}\right)\right)^{2}}}{\sqrt{2 \pi}} .
$$

Note that $K_{c b s}$ depends only on the cost parameters $c, b$ and $s$.

\subsection{Optimal Forecast Precision in the Cooperative Case}

We now examine the optimal forecast precisions, or equivalently, the optimal standard deviations. Since we assume that the price function of the forecast generation is the same at every agent $\left(p\left(\sigma_{i}\right)=\alpha / \sigma_{i}^{2}\right)$, it follows that in the optimal solution each $\sigma_{i}$ is the same $\left(\sigma_{i}=\sigma_{j}, \forall i, j \in[1, n]\right)$ and therefore $\sigma=\sqrt{\sum_{i=1}^{n} \sigma_{i}^{2}}=\sqrt{n} \sigma_{i}$.

The utility will be the valuation minus the forecasting price of the $n$ agents:

$$
U\left(\sigma_{i}\right)=v^{*}(\sigma)-n p\left(\sigma_{i}\right)=-c m-\sqrt{n} \sigma_{i} K_{c b s}-n \frac{\alpha}{\sigma_{i}^{2}} .
$$

This utility function is concave too $\left(U^{\prime \prime}\left(\sigma_{i}\right)=-6 n \alpha /\left(\sigma_{i}\right)^{4} \leq 0\right)$, therefore the optimal standard deviation can be determined by

$$
U^{\prime}\left(\sigma_{i}^{*}\right)=2 n \alpha /\left(\sigma_{i}^{*}\right)^{3}-\sqrt{n} K_{c b s}=0,
$$

which results in

$$
\sigma_{i}^{*}=\sqrt[3]{\frac{2 \sqrt{n} \alpha}{K_{c b s}}} .
$$

The optimal forecast precision in function of the number of the home agents is illustrated on Fig. 2.

\section{Non-Cooperative Mechanism}

In this section we consider a non-cooperative setting, where each agent maximises its own utility. A home agent has two decisions: about the precision of the generated forecast $\left(\sigma_{i}\right)$, and the values of the reported forecast $\left(\hat{m}_{i}, \hat{\sigma}_{i}\right)$, while the aggregator decides about the purchased quantity $(q)$. The mechanism in this case is characterised by a transfer function or payment $t_{i}\left(\hat{m}_{i}, \hat{\sigma}_{i}, \xi_{i}\right)$ that has to be paid by agent $i$ to the aggregator after the consumption $\xi_{i}$ is realised. The transfer function should depend only on commonly known parameters and independent from the privately known $m_{i}$ and $\sigma_{i}$.

We are looking for a mechanism that is realized by such a transfer function that fulfils the following four key properties: 
- It has incentive compatibility that implies in this case two things: (i) the home agents create optimally precise forecasts, and (ii) they report the forecasts to the aggregator truthfully, if they want to maximise their utility.

- The mechanism is efficient, i.e., the aggregator agent purchases the optimal quantity of electricity from the market.

- The mechanism is individually rational, i.e., the utility of the home agents are not less than their utility without the mechanism (when purchasing directly from the market).

- Finally, it is budget balanced meaning that the mechanism does not run into deficit or surplus, or in other words, the utility of the aggregator is zero.

We assume that the market prices $c, b$ and $s$ are common knowledge, since the home agents can buy directly from the market if they do not join the mechanism. We suggest the same transfer function that we developed for coordinating supply chains, that consists of two terms: (i) the payment for the purchased electricity, and (ii) a penalty for the forecast error, based on a scoring rule [10]:

$$
t_{i}\left(\hat{m}_{i}, \hat{\sigma}_{i}, \xi_{i}\right)=c \xi_{i}+\gamma\left(\frac{\left(\xi_{i}-\hat{m}_{i}\right)^{2}}{\hat{\sigma}_{i}}+\hat{\sigma}_{i}\right),
$$

where $\hat{m}_{i}$ and $\hat{\sigma}_{i}$ are the communicated forecast of agent $i, \xi_{i}$ is its realised consumption, while $\gamma$ is a positive constant. As we shall soon see, in order to achieve the four required properties stated above, $\gamma$ cannot be arbitrary, but it should assume a specific value.

It is worth noting that the payment (10) is very similar to the classical logarithmic scoring rule, which can be determined as the negated logarithm of the probability density function of the communicated forecast, i.e., in case of the normal distribution, it is the following

$$
\frac{\ln 2 \pi}{2}+\left(\frac{\left(\xi_{i}-\hat{m}_{i}\right)^{2}}{2 \hat{\sigma}_{i}^{2}}+\ln \hat{\sigma}_{i}\right) .
$$

It is well known that a positive affine transformation of a strictly proper scoring rule remains strictly proper [4], but in spite of the similarities, these two scoring rules are not affine transformations of each other.

In what follows we examine one by one whether and how the non-cooperative mechanism exhibits the basic properties required above.

\subsection{Incentive Compatibility}

In the first phase the home agents generate forecasts $m_{i}$ and $\sigma_{i}$, next, they report the forecasts to the aggregator agent in the centre. Let us first examine the latter phase with generated forecast $\left(m_{i}, \sigma_{i}\right)$, reported forecast $\left(\hat{m}_{i}, \hat{\sigma}_{i}\right)$, and realized demand $\xi_{i}$. Note that at this point since $p\left(\sigma_{i}\right)$ has been already invested in the forecast, we consider only the expected payment here.

Theorem 1. The unique optimal solution for minimising the expected payment is $\hat{m}_{i}=m_{i}$ and $\hat{\sigma}_{i}=\sigma_{i}$, therefore the home agents are inspired to report the forecasts truthfully. 
Proof. The expected payment is

$$
\begin{aligned}
\mathbb{E}\left[t_{i}\left(\hat{m}_{i}, \hat{\sigma}_{i}, \xi_{i}\right)\right] & =c \mathbb{E}\left[\xi_{i}\right]+\gamma \mathbb{E}\left[\frac{\left(\xi_{i}-\hat{m}_{i}\right)^{2}}{\hat{\sigma}_{i}}+\hat{\sigma}_{i}\right] \\
& =c m_{i}+\gamma \mathbb{E}\left[\frac{\xi_{i}^{2}+\hat{m}_{i}^{2}-2 \hat{m}_{i} \xi_{i}}{\hat{\sigma}_{i}}+\hat{\sigma}_{i}\right] \\
& =c m_{i}+\gamma \frac{\mathbb{E}\left[\xi_{i}^{2}\right]+\hat{m}_{i}^{2}-2 \hat{m}_{i} \mathbb{E}\left[\xi_{i}\right]}{\hat{\sigma}_{i}}+\hat{\sigma}_{i} \\
& =c m_{i}+\gamma\left(\frac{m_{i}^{2}+\sigma_{i}^{2}+\hat{m}_{i}^{2}-2 \hat{m}_{i} m_{i}}{\hat{\sigma}_{i}}+\hat{\sigma}_{i}\right),
\end{aligned}
$$

where we have applied the identity $\mathbb{E}\left[\xi_{i}^{2}\right]=m_{i}^{2}+\sigma_{i}^{2}$. The partial derivative of the expected payment by $\hat{m}_{i}$ is

$$
\frac{\partial \mathbb{E}\left[t_{i}\left(\hat{m}_{i}, \hat{\sigma}_{i}, \xi_{i}\right)\right]}{\partial \hat{m}_{i}}=\gamma\left(\frac{2 \hat{m}_{i}-2 m_{i}}{\hat{\sigma}_{i}}\right),
$$

which equals zero iff $\hat{m}_{i}=m_{i}$, independently from the value of $\hat{\sigma}_{i}$. This yields the minimum, since the expected payment is convex in $\hat{m}_{i}$ :

$$
\frac{\partial^{2} \mathbb{E}\left[t_{i}\left(\hat{m}_{i}, \hat{\sigma}_{i}, \xi_{i}\right)\right]}{\partial \hat{m}_{i}^{2}}=\gamma\left(\frac{2}{\hat{\sigma}_{i}}\right) \geq 0 .
$$

For calculating the other partial derivative, we already exploit that $\hat{m}_{i}=m_{i}$ :

$$
\frac{\partial \mathbb{E}\left[t_{i}\left(m_{i}, \hat{\sigma}_{i}, \xi_{i}\right)\right]}{\partial \hat{\sigma}_{i}}=\gamma\left(-\frac{\sigma_{i}^{2}}{\hat{\sigma}_{i}^{2}}+1\right),
$$

which equals zero iff $\hat{\sigma}_{i}=\sigma_{i}$, which is the minimum, since the expected payment is convex also in $\hat{\sigma}_{i}$ :

$$
\frac{\partial^{2} \mathbb{E}\left[t_{i}\left(m_{i}, \hat{\sigma}_{i}, \xi_{i}\right)\right]}{\partial \hat{\sigma}_{i}^{2}}=\gamma \frac{\sigma_{i}^{2}}{\hat{\sigma}_{i}^{3}} \geq 0
$$

Let us now examine the first phase knowing that later the forecasts will be reported truthfully. In this case, the expected payment can be derived from (12): $\mathbb{E}\left[t_{i}\left(m_{i}, \sigma_{i}, \xi_{i}\right)\right]=c m_{i}+2 \gamma \sigma_{i}$, thus the utility of agent $i$ becomes:

$$
U_{i}\left(\sigma_{i}\right)=-\mathbb{E}\left[t_{i}\left(m_{i}, \sigma_{i}, \xi_{i}\right)\right]-p\left(\sigma_{i}\right)=-c m_{i}-2 \gamma \sigma_{i}-\frac{\alpha}{\sigma_{i}^{2}} .
$$

Since the utility is concave in $\sigma_{i}\left(U_{i}^{\prime \prime}\left(\sigma_{i}\right)=-6 \alpha /\left(\sigma_{i}\right)^{4} \leq 0\right)$, the optimal standard deviation can be determined by $U_{i}^{\prime}\left(\sigma_{i}^{*}\right)=-2 \gamma+2 \alpha /\left(\sigma_{i}^{*}\right)^{3}=0$, which yields

$$
\sigma_{i}^{*}=\sqrt[3]{\frac{\alpha}{\gamma}}
$$

therefore using $\gamma=\frac{K_{c b s}}{2 \sqrt{n}}$ results in the the optimal forecast precision of (9). 


\subsection{Efficiency}

The utility of the aggregator will be the collected transfer payments minus the price of the electricity bought, plus the price of the electricity eventually soldthis last two items are summed up by the valuation (1), i.e.,

$$
U_{a}(q)=\mathbb{E}\left[\sum_{i=1}^{n} t_{i}\left(m_{i}, \sigma_{i}, \xi_{i}\right)\right]+v(q) .
$$

Since the first term is independent from the decision variable $q$, thus the aggregator intends to maximise the valuation that results in the optimal quantity derived in (3). Hence, the system as a whole meets the demand of home agents in expectation at the lowest possible total price of electricity.

\subsection{Individual Rationality}

Without the mechanism, home agent $i$ would generate its forecast and buy the electricity directly from the forward and balancing markets. Now we investigate if any home agent $i$ could be better off without joining to the mechanism. Let $q_{i}$ denote the quantity bought on the forward market, then analogously to the derivation of Section 3.1, its revenue become $-c q_{i}-b \max \left(\xi_{i}-q_{i}, 0\right)+s \max \left(q_{i}-\right.$ $\left.\xi_{i}, 0\right)$, and its expected value

$$
v_{i}\left(q_{i}\right)=-c q_{i}+b\left(q_{i}-m_{i}\right)+(b-s)\left(\int_{-\infty}^{q_{i}} x \phi_{i}(x) d x-q_{i} \Phi_{i}\left(q_{i}\right)\right),
$$

where $\phi_{i}$ and $\Phi_{i}$ are the probability and cumulative distribution functions of $\xi_{i}$, respectively. This yields an optimal purchase quantity

$$
q_{i}^{*}=m_{i}+\sigma_{i} \sqrt{2} \operatorname{erf}^{-1}\left(\frac{b-2 c+s}{b-s}\right),
$$

and

$$
v_{i}^{*}\left(\sigma_{i}\right):=v_{i}\left(q_{i}^{*}\right)=-c m_{i}-\sigma_{i} K_{c b s} .
$$

Then the optimal forecast precision can be derived analogously to Section 3.2 using

$$
\begin{gathered}
\bar{U}_{i}\left(\sigma_{i}\right)=v_{i}^{*}\left(\sigma_{i}\right)-p\left(\sigma_{i}\right)=-c m_{i}-\sigma_{i} K_{c b s}-\frac{\alpha}{\sigma_{i}^{2}}: \\
\bar{\sigma}_{i}^{*}=\sqrt[3]{\frac{2 \alpha}{K_{c b s}}} .
\end{gathered}
$$

All in all, the utility of agent $i$ without the mechanism is

$$
\begin{aligned}
\bar{U}_{i}\left(\bar{\sigma}_{i}^{*}\right)=v_{i}^{*}\left(\bar{\sigma}_{i}^{*}\right)-p\left(\bar{\sigma}_{i}^{*}\right) & =-c m_{i}-\bar{\sigma}_{i}^{*} K_{c b s}-p\left(\bar{\sigma}_{i}^{*}\right) \\
& =-c m_{i}-\frac{3}{\sqrt[3]{4}} \sqrt[3]{\alpha K_{c b s}^{2}}
\end{aligned}
$$


However, by using the mechanism (9) and (17) are valid, thus

$$
\begin{aligned}
U_{i}\left(\sigma_{i}^{*}\right) & =-\mathbb{E}\left[t_{i}\left(m_{i}, \sigma_{i}^{*}, \xi_{i}\right)\right]-p\left(\sigma_{i}^{*}\right) \\
& =-c m_{i}-\frac{3}{\sqrt[3]{4 n}} \sqrt[3]{\alpha K_{c b s}^{2}},
\end{aligned}
$$

and comparing (25) and (26) shows that $U_{i}\left(\sigma_{i}^{*}\right)>\bar{U}_{i}\left(\bar{\sigma}_{i}^{*}\right)$ (if $n>1$ ), i.e., the utility using the mechanism is always greater than without the mechanism. Hence, the home agents have an incentive to use the service of the demand aggregator mechanism when meeting their individual demand for electricity.

\subsection{Budget Balance}

The utility of the aggregator can be computed substituting (3), (5), (9) into (19):

$$
\begin{aligned}
U_{a}\left(q^{*}\right) & =\mathbb{E}\left[\sum_{i=1}^{n} t_{i}\left(m_{i}, \sigma_{i}^{*}, \xi_{i}\right)\right]+v^{*}\left(\sigma^{*}\right) \\
& =n \mathbb{E}\left[t_{i}\left(m_{i}, \sigma_{i}^{*}, \xi_{i}\right)\right]-c m-\sqrt{n} \sigma_{i}^{*} K_{c b s} \\
& =n\left(c m_{i}+2 \gamma \sigma_{i}^{*}\right)-c m-\sqrt{n} \sigma_{i}^{*} K_{c b s} \\
& =c m+2 n \gamma \sigma_{i}^{*}-c m-\sqrt{n} \sigma_{i}^{*} K_{c b s}=2 n \gamma \sigma_{i}^{*}-\sqrt{n} \sigma_{i}^{*} K_{c b s} \\
& =2 n \frac{K_{c b s}}{2 \sqrt{n}} \sqrt[3]{\frac{2 \sqrt{n} \alpha}{K_{c b s}}}-\sqrt{n} \sqrt[3]{\frac{2 \sqrt{n} \alpha}{K_{c b s}}} K_{c b s}=0,
\end{aligned}
$$

thus the mechanism is ex ante (in expectation) budget balanced. In other words, no payments or debts are accumulated at the aggregator agent.

\section{Computational Study}

In this section we illustrate the properties of the mechanism on a specific numerical example and simulation runs. We apply the same experimental set-up as in [9], i.e., we set $c=100, b=170, s=50$ and $\alpha=20$. Fig. 2 shows the optimal forecast precision as the number of home agents increases. It can be observed that as more and more agents join the mechanism, the required precision - thus the forecasting cost - considerably decreases.

Fig. 3 plots the expected and the simulated average utility of the home agents, where the expected demand $\left(m_{i}\right)$ for each agent is uniformly distributed in the interval $[30,50]$. Each value is an average of 1000 simulation runs, while the expected cost curve is given by $(26)$.

The previous two figures point out that the largest marginal gain joining the mechanism is achieved when there are only few agents in the mechanism already. This means that although joining a larger coalition is always better than joining a smaller, but only slightly. In other words, a lot of independent mechanisms with relatively small number of homes are almost as economical as a single mechanism with all the agents. It is an interesting property that a 


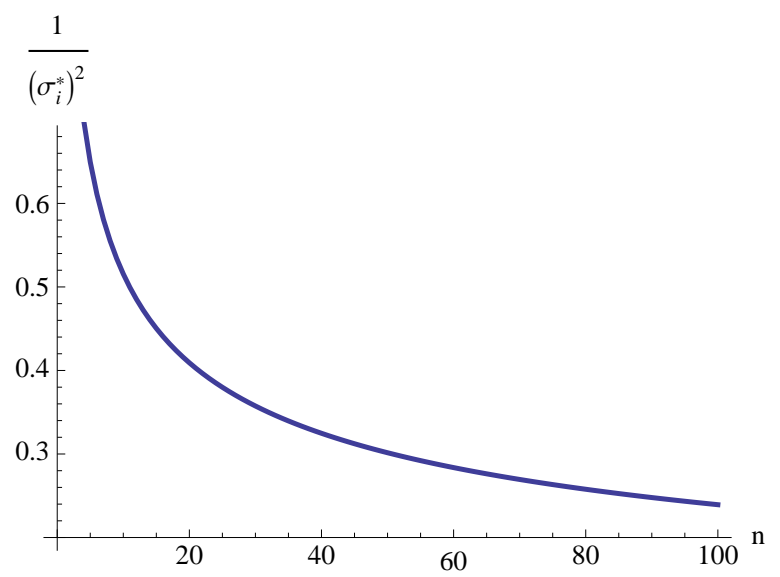

Fig. 2. Required forecast precision.

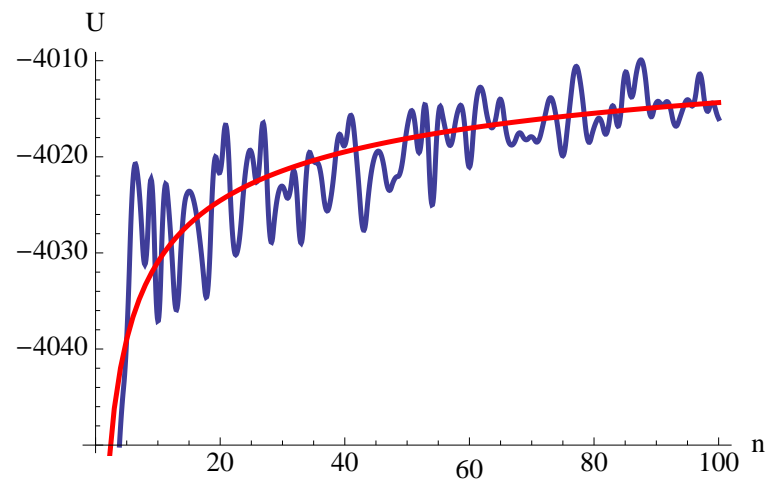

Fig. 3. Average utility of the home agents (simulated and expected).

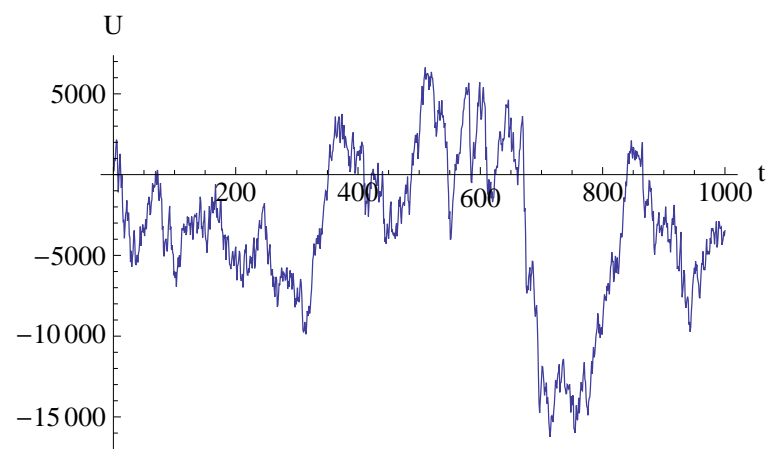

Fig. 4. Cumulated utility of the aggregator with 100 agents as time progresses. 
mechanism with more home agents can achieve better performance, even when the forecasts are less precise.

Fig. 4 shows the simulated cumulative utility of the aggregator agent. Although its expected value is zero as it was proved, its realised value can be arbitrary, oscillating around zero.

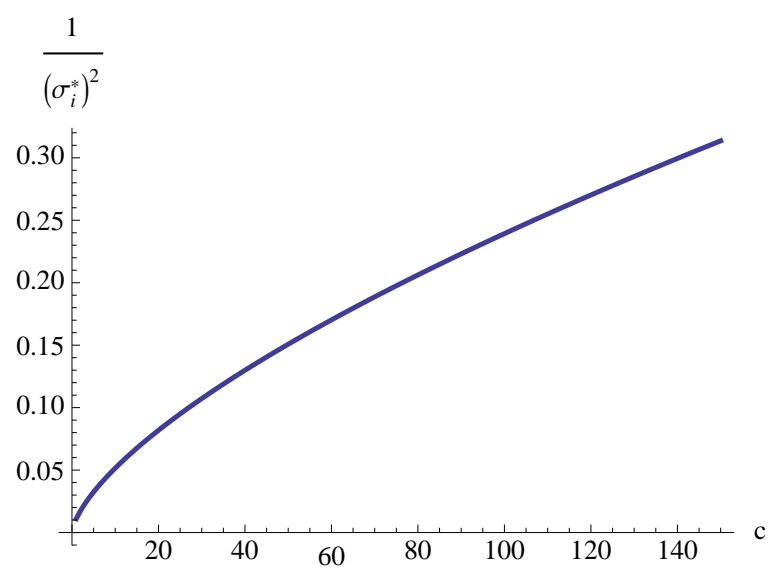

Fig. 5. Required forecast precision as cost parameters increase.

In a smart grid the cost parameters can change from hour to hour, therefore it is important to study the behaviour of the system under different costs. In the next experiment we fix $n=100$ and the ratios $b / c=1.7$ and $c / s=2$, therefore the cost parameters change proportionately. Fig. 5 illustrates that as the purchasing costs increase, it becomes more important to possess more precise forecasts. In addition, it can be noted that if the ratio between the cost parameters is fixed, the $K_{c b s}$ value is linear in $c$ (see (6)), and thus $\gamma$ too.

\section{Conclusions and Future Works}

This paper investigated a mechanism design problem in a setting where several agents can generate estimates of independent future demands at a cost. An aggregator agent elicits the forecasts and based on this information, optimises a procurement decision. A novel scoring rule based mechanism has been developed which is incentive compatible, efficient, individually rational and ex ante budget balanced, contrary to prior works in this field. Furthermore, the proposed mechanism respects privacy, since forecasts and sensitive consumption data have to be known only by the affected home agent and the aggregator. Several simulation runs confirmed that even a small group of agents can significantly increase their utility by forming such a mechanism compared to purchasing directly from the market. Further on, a relatively small group can achieve almost as much benefit as a larger one. 
A practical extension of the presented model is when each agent has different $\alpha_{i}$ values. In this case calculating the optimal forecast precisions leads to a nonlinear optimisation problem with $n$ variables. Consequently, it may be necessary to apply different $\gamma_{i}$ parameters for the home agents, and for this purpose, the $\alpha_{i}$ parameters have to be common knowledge. From (18) with an arbitrary $\gamma$ these parameters can be calculated as $\alpha_{i}=\gamma\left(\sigma_{i}^{*}\right)^{3}$, thus cost information can be elicited after an initial step. However, the above derivation is only a sketch and needs further analysis.

Acknowledgments. This work has been supported by the KMR_12-1-20120031 (E+Grid) grant and by the János Bolyai scholarship No. BO/00659/11/6.

\section{References}

1. Katz, R.H., et al.: An information-centric energy infrastructure: The Berkeley view. Sustainable Computing: Informatics and Systems 1(1) (2011) 7-22

2. Egri, P., Váncza, J.: A distributed coordination mechanism for supply networks with asymmetric information. Eur. J. of Op. Res. 226(3) (2013) 452-460

3. Apt, K.: A primer on strategic games. In Apt, K., Graedel, E., eds.: Lectures in Game Theory for Computer Scientists. Cambridge University Press (2011) 1-37

4. Zohar, A., Rosenschein, J.S.: Mechanisms for information elicitation. Artificial Intelligence 172(16-17) (2008) 1917-1939

5. Chen, Y., Pennock, D.M.: Designing markets for prediction. AI Magazine 31(4) (2010) 42-52

6. Papakonstantinou, A., Rogers, A., Gerding, E., Jennings, N.: Mechanism design for the truthful elicitation of costly probabilistic estimates in distributed information systems. Artificial Intelligence 175(2) (2011) 648-672

7. Blumsack, S., Fernandez, A.: Ready or not, here comes the smart grid! Energy 37(1) (2012) 61-68

8. Jackson, J.: Improving energy efficiency and smart grid program analysis with agent-based end-use forecasting models. Energy Policy 38(7) (2010) 3771-3780

9. Rose, H., Rogers, A., Gerding, E.H.: A scoring rule-based mechanism for aggregate demand prediction in the smart grid. In: 11th International Conference on Autonomous Agents and Multiagent Systems (AAMAS 2012). (2012) 661-668

10. Egri, P., Váncza, J.: Supply network coordination by vendor managed inventory - a mechanism design approach. In: Proc. of the 2nd Workshop on Artificial Intelligence and Logistics (AILog-2011), 22nd International Joint Conference on Artificial Intelligence (IJCAI). (2011) 19-24

11. Qin, Y., Wang, R., Vakharia, A.J., Chen, Y., Seref, M.M.: The newsvendor problem: Review and directions for future research. European Journal of Operational Research 213(2) (2011) $361-374$

12. Ketzenberg, M.E., Rosenzweig, E.D., Marucheck, A.E., Metters, R.D.: A framework for the value of information in inventory replenishment. European Journal of Operational Research 182(3) (2007) 1230-1250

13. Oliveira, F.S., Ruiz, C., Conejo, A.J.: Contract design and supply chain coordination in the electricity industry. Eur. J. of Op. Res. 227(3) (2013) 527-537

14. Chalkiadakis, G., Robu, V., Kota, R., Rogers, A., Jennings, N.: Cooperatives of distributed energy resources for efficient virtual power plants. In: 10th Int, Conf. on Autonomous Agents and Multiagent Systems (AAMAS-2011). (2011) 787-794 